\title{
GENERIC SUBMANIFOLDS OF A LOCALLY CONFORMAL KAEHLER MANIFOLD-II
}

\author{
M. HASAN SHAHID \\ Department of Mathematics \\ Faculty of Natural Sciences \\ Jamia Millia Islamia \\ New Delhi-110 025, India \\ and \\ KOUEI SEKIGAWA \\ Department of Mathematics \\ Faculty of Science \\ Niigata University \\ Niigata, 950-21, Japan
}

(Received March 6, 1991 and in revised form August 8, 1991)

ABSTRACT. The purpose of this paper is to study generic submanifolds with parallel structures, generic product submanifolds and totally umbilical submanifolds of a locally conformal Kaehler manifold. Moreover, we give some examples of generic submanifolds of a locally conformal Kaehler manifold which are not $C R$-submanifolds.

KEY WORDS AND PHRASES. Locally conformal Kaehler manifold, generic submanifold, $C R$ submanifold.

1991 AMS SUBJECT CLASSIFICATION CODES. 53C35, 53C40.

\section{INTRODUCTION.}

Let $\bar{M}$ be an almost Hermitian. manifold with almost Hermitian structure $(J, g)$. The manifold $\bar{M}$ is called a local conformal Kaehler (briefly, l.c.K.) manifold if for any $x \in \bar{M}$ there is an open neighborhood $थ$ such that, for some differentiable function $\sigma: น \rightarrow R, g^{\prime}=\left.e^{-\sigma} g\right|_{q}$ is a Kaehler metric on $u$. If $u=\bar{M}$ then the manifold is called a globally conformal Kaehler (briefly, g.c.K.) manifold. Let $\Omega$ be the Kaehler form of an almost Hermitian manifold $\bar{M}$ defined by $\Omega(U, V)=g(U, J V)$, for any vector fields $U, V$ on $\bar{M}$. Then it is easy to see that $\bar{M}$ is a l.c.K. manifold if and only if there is a global 1-form $\omega$ (the Lee form of $\bar{M}$ ) such that

$$
d \Omega=\omega \wedge \Omega, \quad d \omega=0,
$$

and $\bar{M}$ is a g.c.K. manifold if and only if $\omega$ is exact. For a l.c.K. manifold $\bar{M}$, the Lee vector field $B$ is given by

$$
g(B, U)=\omega(U)
$$

for any vector field $U$ on $\bar{M}$. We denote by $\bar{\nabla}$ the Levi-Civita connection of $g$. We define a torsionfree linear connection $\widetilde{\nabla}$ on $\bar{M}$ by

$$
\tilde{\nabla}_{U} V=\bar{\nabla}_{U} V-\frac{1}{2}\{\omega(U) V+\omega(V) U-g(U, V) B\}
$$


for any vector fields $U, V$ on $\bar{M}$. The linear connection $\widetilde{\nabla}$ is called the Weyl connection of $\bar{M}$. Then we may easily observe that the Weyl connection $\widetilde{\nabla}$ satisfies the condition: $\widetilde{\nabla} J=0, \widetilde{\nabla} g=0$ on each neighborhood on which $\left(J, g^{\prime}=\left.e^{-\sigma} g\right|_{\mathcal{u}}\right)$ is a Kaehler structure.

In general, let $\bar{M}$ be a $2 \mathrm{n}$-dimensional almost Hermitian manifold and $M$ be an m-dimensional Riemman manifold isometrically immersed in $\bar{M}$. Let $\nabla$ be the Levi-Civita connection on $M$ induced by $\bar{\nabla}$. Then the Gauss and Weingarten formulas are given respectively by

$$
\begin{gathered}
\bar{\nabla}_{U} V=\nabla_{U} V+h(U, V), \\
\bar{\nabla}_{U} N=-A_{N} U+\nabla_{U} \frac{1}{} N
\end{gathered}
$$

for any vector fields $U, V$ tangent to $M$ and $N$ normal to $M$, where $h$ is the second fundamental form of $M$ in $\bar{M}$ and $\nabla \perp$ is the normal connection on the normal bundle $T^{\perp}(M)$ with respect to the Levi-Civita connection $\bar{\nabla}$. Then we have $g\left(A_{N} U, V\right)=g(h(U, V), N)$, for any vector fields $U, V$ tangent to $M$. For any vector field $U$ tangent to $M$, we put

$$
J U=P U+F U
$$

where $P U$ and $F U$ are tangential and normal components of $J U$, respectively. Then $P$ is an endomorphism of the tangent bundle $T(M)$ of $M$ and $F$ is a normal bundle valued 1-form on $T(M)$. For any vector field $N$ normal to $M$, we put

$$
J N=t N+f N,
$$

where $t N$ and $f N$ are the tangential and normal components of $J N$, respectively. Then $f$ is an endomorphism of the normal bundle $T^{\perp}(M)$ of $M$ in $\bar{M}$ and $t$ is a tangent bundle valued 1-form on $T^{\perp}(M)$.

DEFINITION: Let $M$ be a submanifold of an almost Hermitian manifold $\bar{M}$. The holomorphic subspace $D_{x}$ of $T_{x} M$ at $x \in M$ is defined by $D_{x}=T_{x} M \cap J T_{x} M . \quad D_{x}$ is the maximal complex subspace of $T_{x} \bar{M}$ which is contained in $T_{x} M$. If the dimension of $D$ is constant along $M$, and furthermore, $D$ defines a differentiable distribution on $M$, then $M$ is called a generic submanifold of $\bar{M}$.

Let $M$ be a generic submanifold of an almost Hermitian manifold $\bar{M}$. We call the distribution $D$ the holomorphic distribution and the orthogonal complementary distribution $D^{\perp}$ the purely real distribution. They satisfy the following relations:

$$
D_{x} \cap D_{x}^{\perp}=\{0\}, \quad D_{x}^{\perp} \cap J D_{x}^{\perp}=\{0\} \text { for each } x \in M .
$$

Let $\nu_{x}$ be the holomorphic normal space of $M$ at $x$, i.e.,

$$
\nu_{x}=T_{x}^{\perp} M \cap J T_{x}^{\perp} M .
$$

Then $\nu_{x}(x \in M)$ defines a differentiable vector subbundle $\nu$ of $T^{\perp}(M)$ satisfying

$$
T^{\perp}(M)=F D^{\perp}+\nu(\text { direct sum }), t\left(T^{\perp}(M)\right)=D^{\perp} .
$$

Furthermore, we have

$$
D \perp D^{\perp}, P D=D \text { and } D^{\perp} \supset P D^{\perp} \text {. }
$$


We put $\operatorname{dim} D=2 p$ and $\operatorname{dim} D^{\perp}=q$. If $p, q \geq 1$, then the generic submanifold $M$ is said to be proper. In the sequel, we shall consider only proper generic submanifolds. We put

and

$$
\left(\nabla_{U} P\right) V=\nabla_{U}(P U)-P\left(\nabla_{U} V\right)
$$

$$
\left(\nabla_{U} F\right) V=\nabla_{U}^{\frac{1}{U}}(F V)-F \nabla_{U} V
$$

for any vector fields $U, V$ tangent to $M$. We say that $P$ (resp. $F$ ) is parallel if $\left(\nabla_{U} P\right.$ ) $V=0$ (resp. $\left.\left(\nabla_{U} F\right) V=0\right)$ for any vector fields $U, V$ tangent to $M$. If a generic submanifold $M$ of an almost Hermitian manifold $\bar{M}$ satisfies the condition $J D^{\perp} \subset T^{\perp}(M)$, then $M$ is called a $C R$-submanifold of $\bar{M}$. Dragomir ([4]) studied $C R$-submanifolds of l.c.K. manifolds. The present paper is a continuation of the previous work [5].

2. PRELIMINARIES.

Let $M$ be a generic submanifold of a l.c.K. manifold $\bar{M}$. For the Lee vector field $B$ of $\bar{M}$, we put

$$
B=B^{T}+B^{\perp} \text { along } M,
$$

where $B^{T}$ (resp. $B^{\perp}$ ) is the tangential (resp. normal) component of $B$. Furthermore, we put

$$
B^{T}=B^{D}+B^{D \perp} \text { along } M,
$$

where $B^{D}$ (resp. $B^{D^{\perp}}$ ) is the D-component (resp. $D^{\perp}$-component) of $B^{\perp}$. Since $\widetilde{\nabla} J=0$ with respect to the Weyl connection $\widetilde{\nabla}$, taking account of $(1.3) \sim(1.7),(1.11),(1.12),(2.1)$ and $(2.2)$, we have

$$
\begin{aligned}
& \left(\nabla_{X} P\right) Y-\frac{1}{2} \omega(J Y) X+\frac{1}{2} \omega(Y) J X-t h(X, Y) \\
& +\frac{1}{2} g(X, J Y) B^{T}-\frac{1}{2} g(X, Y) P B \perp-\frac{1}{2} g(X, Y) t B^{\perp}=0, \\
& h(X, J Y)-F \nabla_{X} Y+\frac{1}{2} g(X, J Y) B \perp \\
& -\frac{1}{2} g(X, Y) F B^{T}-\frac{1}{2} g(X, Y) f B^{\perp}-f h(X, Y)=0, \\
& \left(\nabla_{X} P\right) Z-A_{F} Z^{X-\frac{1}{2} \omega(J Z) X+\frac{1}{2} \omega(Z) J X-t h(X, Z)=0,} \\
& \left(\nabla_{X} F\right) Z+h(X, P Z)-f h(X, Z)=0 \\
& \left(\nabla_{Z} P\right) X-\frac{1}{2} \omega(J X) Z-\frac{1}{2} \omega(X) P Z-\operatorname{th}(X, Z)=0, \\
& F \nabla_{Z} X-h(J X, Z)+f h(X, Z)=0, \\
& \left(\nabla_{Z} P\right) W-A_{F W} Z-\frac{1}{2} \omega(J W) Z+\frac{1}{2} \omega(W) P Z+\frac{1}{2} g(Z, J W) B^{T} \\
& -\frac{1}{2} g(Z, W) P B^{T}-\frac{1}{2} g(Z, W) t B^{\perp}-t h(Z, W)=0, \\
& \left(\nabla_{Z} F\right) W+h(Z, P W)+\frac{1}{2} g(Z, J W) B^{T}+\frac{1}{2} \omega(W) F Z \\
& -\frac{1}{2} g(Z, W) F B^{T}-\frac{1}{2} g(Z, W) f B^{\perp}-f h(Z, W)=0,
\end{aligned}
$$

for any $X, Y \in D$ and $Z, W \in D^{\perp}$.

We recall the conditions for the distributions $D$ and $D^{\perp}$ to be integrable. 
PROPOSITION 2.1 ([5]). The distribution $D^{\perp}$ is integrable if and only if

$$
g(h(X, J Y)-h(J X, Y)+g(X, J Y) B, F Z)=0,
$$

for any $X, Y \in D$ and $Z \in D^{\perp}$.

PROPOSITION 2.2 ([5]). The distribution $D^{\perp}$ is integrable if and only if

$$
\nabla_{Z}(P W)-\nabla_{W}(P Z)+A_{F} Z^{W-A_{F W}} Z+g(Z, J W) B \in D^{\perp},
$$

for any $Z, W \in D^{\perp}$.

Let $M$ be a totally geodesic generic submanifold of a Kaehler manifold $\bar{M}$. Then it follows immediately that $P$ and $F$ are parallel, and furthermore $D$ is integrable. So, it is worthwhile to study generic submanifolds with parallel structures and also totally umbilical generic submanifolds in a l.c.K. manifold.

\section{GENERIC SUBMANIFOLDS WITH PARELLEL STRUCTURES.}

In this section, we consider generic submanifolds with parallel $P$ (resp. $F$ ) of a l.c.K. manifold.

THEOREM 3.1. Let $M$ be a generic submanifold of a l.c.K. manifold $\bar{M}$. If $P$ is parallel, then $D$ is integrable and $B^{D^{\perp}}=0$ along $M$. Moreover, if $\operatorname{dim} D \geq 4$, then $B^{T}=0$ along $M$.

PROOF. By (1.11) and (2.3), we get

$$
-\frac{1}{2} \omega(J Y) X+\frac{1}{2} \omega(J X) Y+g(X, J Y) B^{T}+\frac{1}{2} \omega(Y) J X-\frac{1}{2} \omega(X) J Y=0,
$$

for $X, Y \in D$. Putting $Y=J X$ in (3.1), we get

$$
\omega(X) X+\omega(J X) J X-g(X, X) B^{\perp}=0,
$$

for any vector field $X$ on $M$. From (3.2), we get

$$
(p-1) g\left(B^{D}, B^{D}\right)+p g\left(B^{D^{\perp}}, B^{D^{\perp}}\right)=0 .
$$

First, we assume $p \geq 2$. Then, by (3.3), we have

$$
B^{D}=0, B^{D^{\perp}}=0\left(\text { and hence } B^{\perp}=0\right) .
$$

Thus, by (2.3) and (3.4), we get

$$
2 \operatorname{th}(X, Y)+g(X, Y) t B=0,
$$

for $X, Y \in D$. On one hand, by (1.11) and (2.4), we get

$$
F \nabla_{X}(P Y)+h(X, Y)+f h(X, J Y)+\frac{1}{2} g(X, Y) B^{\perp}+\frac{1}{2} g(X, J Y) f B^{\perp}=0,
$$

for $X, Y \in D$. By (1.11) and (3.6), we get

$$
F P[X, Y]+f\{h(X, J Y)-h(J X, Y)\}+g(X, J Y) f B^{\perp}=0,
$$

for $X, Y \in D$. From (3.5), we get also

$$
t\{h(X, J Y)-h(J X, Y)\}+g(X, J Y) t B \perp=0,
$$

for $X, Y \in D$. Thus, by (3.7) and (3.8), we have

$$
J\{h(X, J Y)-h(J X, Y)\}+g(X, J Y) J B \perp=-F P[X, Y],
$$

for $X, Y \in D$. By (3.9), we have 


$$
\begin{aligned}
g(h(X, J Y)-h(J X, Y) & +g(X, J Y) B, J Z) \\
& =g(F P[X, Y], Z)=0,
\end{aligned}
$$

for $X, Y \in D$ and $Z \in D^{\perp}$. Thus, from Proposition 3.1 and (3.10), it follows that $D$ is integrable. Next, we assume that $p=1$. Then, by (3.3), we have

By (2.3), we get

$$
B^{D \perp}=0 \text {. }
$$

$$
\begin{aligned}
& \frac{1}{2} \omega(Y) X-\frac{1}{2} \omega(X) Y+\frac{1}{2} \omega(J Y) J X-\frac{1}{2} \omega(J X) J Y \\
& -t\{h(X, J Y)-h(J X, Y)\}-g(X, J Y) P B^{T}-g(X, J Y) t B^{T}=0
\end{aligned}
$$

for $X, Y \in D$. On one hand, by (2.4) and (3.1), we get

$$
F P[X, Y]-f\{h(X, J Y)-h(J X, Y)\}+g(X, J Y) f B^{\perp}=0,
$$

for $X, Y \in D$. By (3.12) and (3.13), we get

$$
\begin{aligned}
J\{h(X, J Y)- & h(J X, Y)\}+g(X, J Y) J B^{T}+g(X, J Y) P B^{T} \\
& +\frac{1}{2} \omega(X) Y-\frac{1}{2} \omega(Y) X+\frac{1}{2} \omega(J X) J Y-\frac{1}{2} \omega(J Y) J X+F P[X, Y]=0,
\end{aligned}
$$

for $X, Y \in D$. From (3.11), it follows that $P B^{T}=J B^{T}$.

Thus, (3.14) implies

$$
\begin{aligned}
h(X, J Y)-h(J X, Y) & +g(X, J Y) B \\
& =\frac{1}{2} \omega(X) J Y-\frac{1}{2} \omega(Y) J X-\frac{1}{2} \omega(J X) Y+\frac{1}{2} \omega(J Y) X+J F P\{X, Y],
\end{aligned}
$$

for $X, Y \in D$. By (3.15), we have

$$
g(h(X, J Y)-h(J X, Y)+g(X, J Y) B, F Z)=g(F P[X, Y], Z)=0,
$$

for $X, Y \in D$ and $Z \in D^{\perp}$. Thus, from (3.16) and Proposition 3.1, if follows that $D$ is integrable.

THEOREM 3.2. Let $M$ be a generic submanifold of a l.c.K. manifold $\bar{M}$ such that $F$ is parallel. Then the distribution $D$ is integrable and each leaf of $D$ is totally geodesic in $M$.

PROOF. By (1.12), we have

$$
0=\left(\nabla_{K} F\right) Y=F \nabla_{X} Y, \text { for } X, Y \in D .
$$

By (3.17), we have $\nabla_{X} Y \in D$ for any $X, Y \in D$, from which the theorem follows immediately.

4. GENERIC PRODUCT SUBMANIFOLDS.

Let $M$ be a generic submanifold of an almost Hermitian manifold $\bar{M}$. If $M$ is locally expressed in the form $M=M_{D} \times M_{D \perp}$, where $M_{D}$ (resp. $M_{D \perp}$ ) is a holomorphic submanifold (resp. a purely real submanifold) of $\bar{M}$, then $M$ is called a generic product submanifold of $\bar{M}$. In this section, we consider generic product submanifold of a l.c.K. manifold $\bar{M}$.

THEOREM 4.1. Let $M$ be a generic product submanifold of a l.c.K. manifold $\bar{M}$. If $B^{D}=0$ along $M$, then we have

and

$$
B^{T}=0 \text { along } M
$$

$$
\nabla_{X} P=0, \quad\left(\nabla_{Z} P\right) X=0
$$

for $X \in D, Z \in D^{\perp}$.

PROOF. Since $\left(\nabla_{X^{P}}\right) Z \in D^{\perp}$, for $X \in D, Z \in D^{\perp}$, by (2.5), we get

$$
g(h(X, Y), F Z)+\frac{1}{2} \omega(J Z) g(X, Y)-\frac{1}{2} \omega(Z) g(J X, Y)=0
$$


for $X, Y \in D, \quad Z \in D^{\perp}$. By (4.3), we get immediately $B^{D^{\perp}}=0$, and hence (4.1). Since $\left(\nabla_{X} P\right) Y \in D$, for $X, Y \in D$, by $(2.3)$ and (4.1), we get

$$
\left(\nabla_{X} P\right) Y=0, \quad \text { for } X, Y \in D .
$$

Since $\left(\nabla_{Z^{P}}\right) \in D^{\perp}$, for $Z, W \in D^{\perp}$, by $(2.9)$ and (4.1), we get

$$
g(h(X, Z), F W)=0, \text { for } X \in D, Z \in D^{\perp} .
$$

by (2.5), (4.1) and (4.5), we have

$$
\begin{aligned}
\mathbf{0} & =g\left(\left(\nabla_{X} P\right) Z, W\right)-g(h(X, W), F Z)-g(t h(X, Z), W) \\
& =g\left(\left(\nabla_{X} P\right) Z, W\right)-g(h(X, W), F Z)+g(h(X, Z), F W) \\
& =g\left(\left(\nabla_{X} P\right) Z, W\right)
\end{aligned}
$$

for $X \in D, Z, W \in D^{\perp}$. By (4.3) and (4.6), we have the first equality of (4.2). Since $\left(\nabla_{Z} P\right) X \in D$, for $X \in D, Z \in D^{\perp}$, by (2.7), we have immediately the second equality of (4.2). $\quad$ Q.E.D.

COROLLARY 4.2. Let $M$ be a $C R$-product submanifold of a 1.c.K. manifold $\bar{M}$. If $B^{D}=0$ along $M$, then $P$ is parallel.

PROOF. Since $P W=0$, and $\nabla_{Z} W,\left(\nabla_{Z} P\right) W \in D^{\perp}$, for $Z, W \in D^{\perp}$, we have immediately $\left(\nabla_{Z} P\right) W=0$ for $Z, W \in D^{\perp}$. Thus, from this together with (4.2), the corollary follows. Q.E.D. 5. TOTALLY UMBILICAL GENERIC SUBMANIFOLDS.

A Riemmannian submanifold $M$ of a Riemannian manifold $\bar{M}$ is called a totally umbilical submanfiold if

$$
h(U, V)=g(U, V) H,
$$

for any vector fields $U, V$ tangent to $M$, where $H$ is the mean curvature vector. In this section, we consider some totally umbilical generic submanifolds of a l.c.K. manifold.

THEOREM 5.1. Let $M$ be a totally umbilical generic submanifold of a l.c.K. manifold $\bar{M}$ such that $P$ is parallel. Then we have $B^{D^{\perp}}=0$ and $2 H+B^{\perp}=0$ along $M$. In particular, if $\operatorname{dim} D \geq 4$. then $2 H+B=0$ along $M$.

PROOF. Since $P$ is parallel, from Theorem 3.1 and (3.4), (3.11), it follows that $D$ is integrable and

By (2.4), we have easily

$$
B^{D \perp}=0 .
$$

$$
2 H+B^{\perp}=0 .
$$

By (3.1), we get

$$
\omega(X)^{2}+\omega(J X)^{2}=g(X, X) g\left(B^{T}, B^{T}\right), \quad \text { for } X \in D .
$$

By (5.2) and (5.4), we have

$$
(p-1) g\left(B^{T}, B^{T}\right)=0 .
$$

By (5.5), if $p \geq 2$, we have $B^{T}=0$. Therefore, the Theorem follows from (5.3).

COROLLARY 5.2. Let $M$ be a totally umbilical generic submanifold of a l.c.K. manifold $\bar{M}$ such that $B \in D$. If $P$ is parallel, then $M$ is totally geodesic and $B=0$ along $M$.

THEOREM 5.3. Let $M$ be a totally umbilical generic submanifold of a l.c.K. manifold $\bar{M}$ such 
that $\operatorname{dim} F D^{\perp}<\operatorname{dim} D^{\perp}$ on a dense open subset in $M$. If $P$ is parallel and $\operatorname{dim} D^{\perp} \geq 2$, then $2 H+B=0$ along $M$.

PROOF. By (1.5), (2.9), (5.1) and (5.2), we have

$$
\begin{aligned}
0= & -\frac{1}{2} \omega(J W) g\left(Z, B^{T}\right)+\frac{1}{2} \omega(J Z) g\left(W, B^{T}\right) \\
& +\frac{1}{2} \omega(W) g\left(P Z, B^{T}\right)-\frac{1}{2} \omega(Z) g\left(P W, B^{T}\right)+g(Z, J W) g\left(B^{T}, B^{T}\right) \\
= & g(Z, J W) g\left(B^{T}, B^{T}\right),
\end{aligned}
$$

for $Z, W \in D^{\perp}$. From (5.2) and (5.6), taking account of Theorem 5.1, the theorem follows immediately.

THEOREM 5.4. Let $M$ be a totally umbilical generic submanifold of a 1.c.K. manifold $\bar{M}$ such that $B \in D^{\perp}$. Then the purely real distribution $D^{\perp}$ is totally geodesic in $\boldsymbol{M}$.

PROOF. For $X \in D, W \in D^{\perp}$ and $N \in T^{\perp}(M)$, by (1.3), (1.5) and (5.1), we have

$$
\begin{aligned}
& 0=g\left(\left(\widetilde{\nabla}_{W^{J}}\right) N, X\right) \\
& =g\left(\widetilde{\nabla}_{W}(J N), X\right)-g\left(J \widetilde{\nabla}_{W} N, X\right) \\
& =g\left(\widetilde{\nabla}_{W}(J N), X\right)+g\left(\bar{\nabla}_{W} N, J X\right) \\
& =g\left(\bar{\nabla}_{W}(t N), X\right)+g\left(\bar{\nabla}_{W}(f N), X\right) \\
& =g\left(\nabla_{W}(t N), X\right),
\end{aligned}
$$

from which the theorem follows immediately.

Q.E.D.

THEOREM 5.5. Let $M$ be a totally umbilical generic submanifold of a l.c.K. manifold $\bar{M}$ such that $F$ is parallel. Then we have $2 H+B \perp=0$ along $M$.

PROOF. Since $F$ is parallel, from Theorem 3.2, it follows that $D^{\perp}$ is integrable and each leaf of $D^{\perp}$ is totally geodesic in $M$. Thus, by (2.4) and (5.1), we have immediately $2 H+B^{\perp}=0$. Q.E.D. 6. EXAMPLES.

In this section, we give some examples of generic submanifolds of Hopf manifolds which are not $C R$-submanifolds. Let $\mathbf{R}^{2 n+2}$ be a $(2 n+2)$-dimensional Euclidean space equipped with the canonical inner product $($,$) and \left\{e_{1}, \ldots, e_{2 n+1}, e_{2 n+2}\right\}$ the canonical orthonormal basis of $\mathbf{R}^{2 n+2}$. We denote by $J_{0}$ the complex structure on $R^{2 n+2}$ defined by

$$
J_{0} e_{2 m-1}=e_{2 m}, J_{0} e_{2 m}=-e_{2 m-1}, \quad 1 \leq m \leq n+1 .
$$

Let $S^{2 n+1}=\left\{x \in \mathbf{R}^{2 n+2} ;(x, x)=1\right\}$ be a $(2 n+1)$-dimensional unit sphere with the canonical Sasakian structure $(\varphi, \xi, \eta, h)$ induced from the Kaehler structure $\left(J_{0},(),\right)$ on $\mathbf{R}^{2 n+2}$. It is well known that the structure vector field $\xi$ defines the Hopf fibration $\pi: S^{2 n+1} \rightarrow C P^{n}$, where $C P^{n}$ is a (complex) $\mathrm{n}$ dimensional complex projective space equipped with the canonical Fibini-Study metric of constant holomorphic sectional curvature 4 . Let $S^{1}=\left\{e^{t \sqrt{-1}} ; t \in \mathbf{R}\right\}$ be a unit circle. We define an almost complex structure $J$ on $M=S^{2 n+1} \times S^{1}$ (resp. $\left.\bar{M}=S^{2 n+1} \times \mathbf{R}\right)$ by

$$
J T=\xi, J \xi=-T \text { and } \quad J U=\varphi U,
$$

for any vector field $U$ on $\bar{M}$ such that $\eta(U)=0$, where $T=\frac{\partial}{\partial t}$ is the canonical unit vector field on $S^{1}$ (resp. $\left.\mathbf{R}^{1}\right)$. Then $\left(S^{2 n+1} \times S^{1}, J\right)$ (resp. $\left.\left(S^{2 n+1} \times \mathbf{R}^{1}, J\right)\right)$ is a l.c.K. manifold (resp. a g.c.K. manifold) together with the product metric $g=h+1$ on $\bar{M}=S^{2 n+1} \times S^{1}\left(\right.$ resp. $\left.\bar{M}=S^{2 n+1} \times \mathbf{R}^{1}\right)$. Then the Lee form $\omega$ of $\bar{M}$ is given by $\omega=2 d t$.

I. We denote by $S_{p q}$ the Segre imbedding $S_{p q}: \mathrm{C} P^{p} \times \mathrm{C} P^{q} \rightarrow \mathrm{C} P^{p+q+p q}([2])$. Let $M_{1}$ be any qdimensional purely real submanifold of $C P^{q}$. Then $M=C P^{p} \times M_{1}$ is a generic product submanifold 
of $C P^{p+q+p q}$ in which $C P^{p}$ is imbedded as a totally geodesic complex submanifold. We denote by the immersion $\iota: M_{1} \rightarrow C P^{q}$. Let $M=\left\{S_{p q} \circ(1 \times \iota)^{-1}\left(S^{2(p+q+p q)+1}\right)\right.$ be pull-back of the Hopf bundle $\left.\pi: S^{2(p+q+p q)+1}\right)$ by the immersion $S_{p q} \circ(1 \times \iota): C P^{p} \times M_{1} \rightarrow C P^{p+q+p q}$. Then we may easily observe that $M$ is a generic submanifold of the Hopf manifold $\bar{M}=S^{2(p+q+p q)+1} \times S^{1}$. For example, let $M_{1}$ be the real submanifold of $C P^{q}(q>1)$ defined by

$M_{1}=\left\{\left(x_{0}, \ldots, x_{q-1}, x_{q}+\sqrt{-1} x_{q-1}\right) \in \mathbf{C} P^{q} ; \quad\left(x_{0}, \ldots, x_{q-1}, x_{q}\right)\right.$ are homogeneous coordinates of a $\mathrm{q}-$ dimensional real projective space $R P^{q}$ \}. Then $M_{1}$ is a purely real submanifold of $C P^{q}$ which is not totally real.

In the following II $\sim$ IV, we assume that $\bar{M}=S^{7} \times S^{1}$.

II. Let $\Pi$ be the 5-dimensional linear subspace of $\mathbf{R}^{8}$ given by $\Pi=\operatorname{span}_{\mathbf{R}}\left\{e_{1}, \ldots, e_{5}\right\}$. We put $S^{4}=S^{7} \cap \Pi$ and $M_{2}^{4}=\left\{x=\sum_{i=1}^{5} x_{i} e_{i} \in S^{4} ; 0<x^{5}<1\right\}$. For each point $x \in M_{2}^{4}$, let $D_{x}^{\prime}$ be the subspace of $T_{x} M_{2}^{4}$ defined by $D_{x}^{\prime}=\left\{u \in T_{x} M_{2}^{4} ;\left(u, J_{0} x\right)=0,\left(u, e_{5}\right)=0\right\}$. We put $M=M_{2}^{4} \times S^{1}\left(\subset S^{7} \times S^{1}\right)$. For each point $\left(x, e^{\sqrt{-1} t}\right) \in M$, let $D_{\left(x, e^{\sqrt{-1} t}\right)}$ be the subspace of $T\left(x, e^{\sqrt{-1} t}\right){ }^{M}$ defined by $D_{\left(x, e^{\sqrt{-1} t}\right)}=\left\{(u, 0) \in T\left(x, e^{\sqrt{-1} t}\right)\right.$ M $\left.u \in D_{x}^{\prime}\right\}$. Then we may easily observe that $M$ is a totally geodesic generic submanifold of $\bar{M}$ with the holomorphic distribution $D$ which is not a $C R$ submanifold of $\bar{M}$. We may easily check that the Lee form of $\bar{M}$ is tangent to $M$.

III. We put $M=M_{2}^{4} \times\{1\}\left(\subset S^{7} \times S^{1}\right)$. Then $M$ is also a totally geodesic generic submanifold of $\bar{M}$ with holomorphic distribution $D$ as in II (restricted to $M_{2}^{4} \times\{1\}$ ) which is not $C R$-submanifold of $\bar{M}$. In this case, we may easily check that the Lee form of $\bar{M}$ is normal to $\boldsymbol{M}$.

IV. We put $M_{3}^{4}=\left\{x=\sum_{i=1}^{5} x_{i} e_{i}+\frac{1}{\sqrt{2}} e_{7} \in S^{7} ; 0<x_{5}<\frac{1}{\sqrt{2}}\right\}$. For each point $x \in M_{3}^{4}$, let $D_{x}^{\prime \prime}$ be the subspace of $T_{x} M_{3}^{4}$ defined by $D_{x}^{\prime \prime}=\left\{u \in T_{x} M_{3}^{4} ;\left(u, J_{0} x\right)=0,\left(u, e_{5}\right)=0\right\}$. We put $M=M_{3}^{4} \times\{1\}$. For each point $(x, 1) \in M$, let $D_{(x, 1)}$ be the subspace of $T_{(x, 1)} M$ defined by $D_{(x, 1)}=\left\{(u, 0) \in T(x, 1)\right.$; $\left.u \in D_{x}^{\prime \prime}\right\}$. Then we may easily observe that $M$ is a totally umbilical generic submanifold of $\bar{M}$ with holomorphic distribution $D$ which is not a $C R$-submanifold of $\bar{M}$ and is not totally geodesic in $\bar{M}$.

ACKNOWLEDGEMENT. The authors would like to express their hearty thanks to Professor L. Vanhecke for his kind advices.

\section{REFERENCES}

1. BEJANCU, A., CR-submanifolds of a Kaehler manifold I, Proc. Amer. Math. Soc. 69 (1978), 135-142.

2. CHEN, B.Y., Geometry of Submanifolds, New York, 1973.

3. CHEN, B.Y., Differential geometry of real submanifolds in a Kaehler manifold, Monatsh. Math. 91 (1981), 257-274.

4. DRAGOMIR, S., Cauchy-Rieman submanifolds of locally conformal Kaehler manifold, Geometriae Dedicata 28 (1988), 181-197.

5. SHAHID, M.H. \& HUSAIN, S.I., Generic submanifolds of a locally conformal Kaehler manifold, Soochow J. Math. 14 (1983), 111-117.

6. KASHIWADA, T., Some properties of locally conformal Kaehler manifolds, Hokkaido Math. J. 8 (1979), 191-198.

7. VAISMAN, I., On locally and globally conformal Kaehler manifolds, Trans, Amer. Math. Soc. $\underline{262}(1980), 533-542$. 


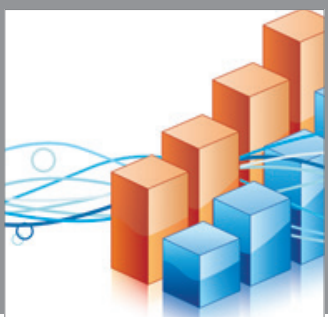

Advances in

Operations Research

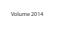

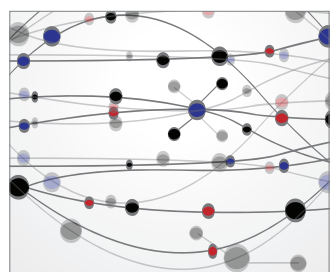

\section{The Scientific} World Journal
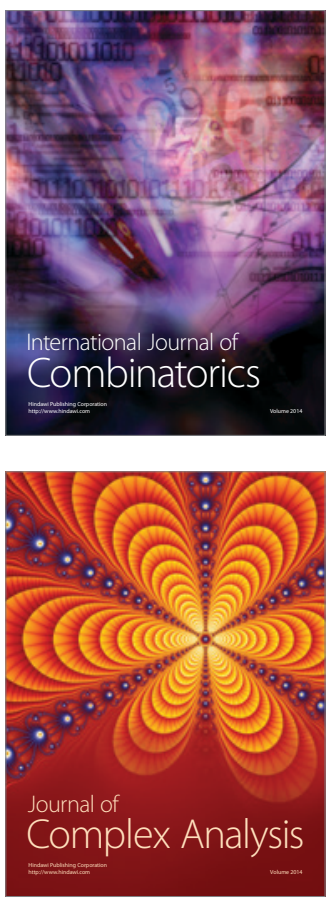

International Journal of

Mathematics and

Mathematical

Sciences
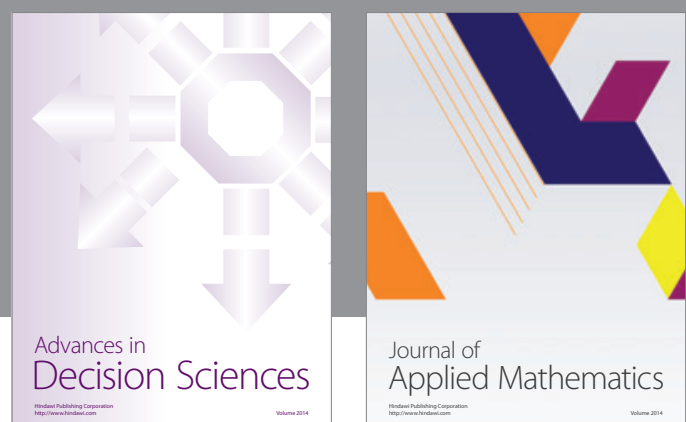

Journal of

Applied Mathematics
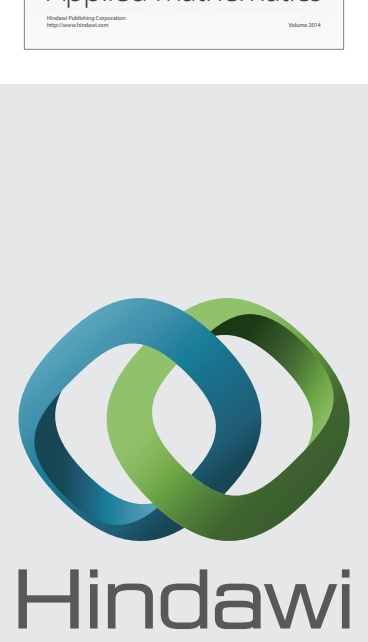

Submit your manuscripts at http://www.hindawi.com
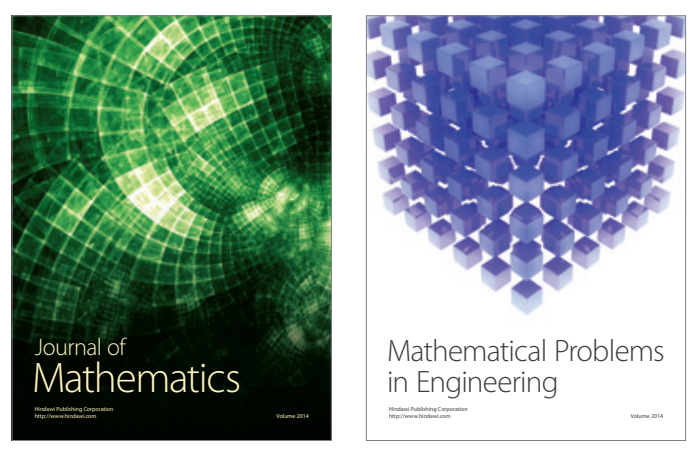

Mathematical Problems in Engineering
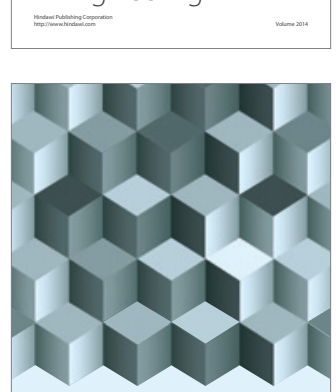

Journal of

Function Spaces
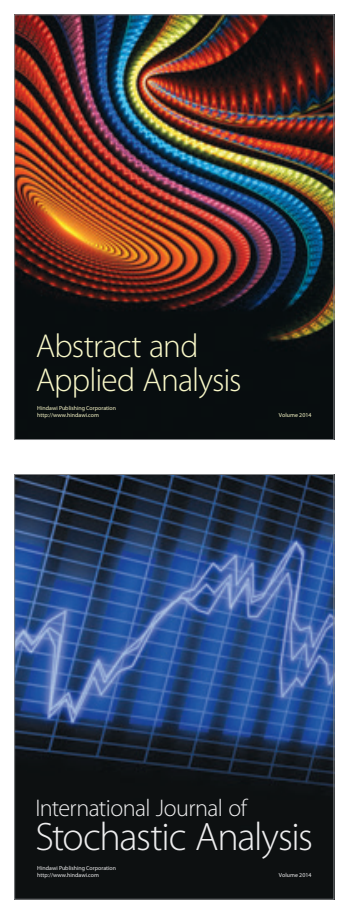

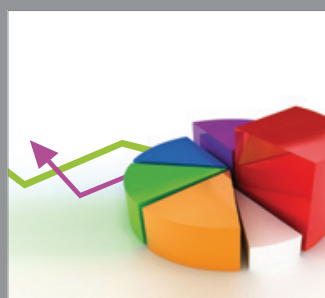

ournal of

Probability and Statistics

Promensencen
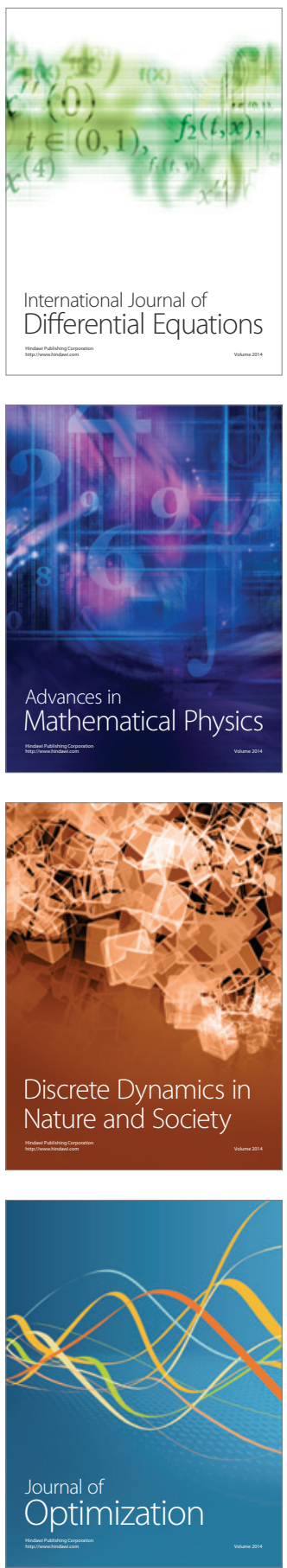